\title{
How children's glue fixes a decades old enigma
}

\author{
Michael A. Rieger ${ }^{1,2,3}$ \\ ${ }^{1}$ Department of Medicine, Hematology/Oncology, Goethe University Hospital, Frankfurt, Germany; ${ }^{2}$ German Cancer Consortium (DKTK) and \\ German Cancer Research Center (DKFZ), Heidelberg, Germany; ${ }^{3}$ Frankfurt Cancer Institute, Frankfurt, Germany \\ Correspondence to: Michael A. Rieger, PhD. Department of Medicine II, Hematology/Oncology, Goethe University Hospital, Theodor-Stern-Kai 7, \\ 60590 Frankfurt am Main, Germany. Email: m.rieger@em.uni-frankfurt.de. \\ Provenance: This is an invited article commissioned by the Section Editor Dr. Liuhua Zhou (Department of Urology, Nanjing First Hospital, Nanjing \\ Medical University, Nanjing, China). \\ Comment on: Wilkinson AC, Ishida R, Kikuchi M, et al. Long-term ex vivo haematopoietic-stem-cell expansion allows nonconditioned \\ transplantation. Nature 2019;571:117-21.
}

Submitted Sep 03, 2019. Accepted for publication Sep 16, 2019.

doi: 10.21037/atm.2019.09.90

View this article at: http://dx.doi.org/10.21037/atm.2019.09.90

Bone marrow and stem cell transplantations have become a life-saving treatment for millions of patients suffering from hematologic disorders and cancer (1). Long-term repopulating hematopoietic stem cells (LT-HSCs) in the graft of the donor facilitate the life-long blood cell reconstitution in the recipient (2). LT-HSCs are multipotent adult stem cells that have the potential to regenerate all blood cell lineages (3). They are collected from adult bone marrow and umbilical cord blood as well as from peripheral blood after mobilization from the bone marrow. While stem cell transplantations have become a prime example for successful cell therapy and regenerative medicine, the treatment regime still bears several challenges. First, in allogeneic transplantations of a graft from an unrelated donor, an HLA-matching donor must be identified and severe graft-versus-host reactions must be avoided. This requires a tremendous logistic and financial effort and mainly covers only HLA-haplotypes of large ethnical groups. Second, a fast blood cell recovery is influenced by the number of functional stem cells in the graft. Especially in umbilical cord blood, a source of very potent LT-HSCs, the number of stem cells is insufficient for an adult recipient and cord blood units of multiple donors must be combined. Third, gene therapy approaches require several days of in vitro culture to genetically repair mutations in LT-HSCs of patients with inherited hematologic disorders, and the potency of LT-HSCs rapidly and successively decreases over time. Also, the number of repaired LT-HSCs dictate the success of the treatment, especially in disorders where the gene correction does not provide a selective advantage for the repaired LT-HSCs.

LT-HSCs reside in the bone marrow in a deeply quiescent status, therefore they are out of the cell cycle and rarely divide during lifetime (4). The microenvironment in the bone marrow niche appears vital for the preservation of LT-HSCs biology with its distinct metabolic properties $(5,6)$. Although the prospective isolation of LT-HSCs from bone marrow or cord blood using multiple surface markers via flow cytometry-based cell sorting has been established for a few decades $(7,8)$, the maintenance of LTHSCs in in vitro culture has still not been achieved yet (9). Once retrieved from their environmental niche in the bone marrow and taken in culture, LT-HSCs spontaneously and immediately start to differentiate and successively lose their stem cell potential within a few days (10). When LTHSCs divide they either self-renew to preserve their stem cell identity or they differentiate leading to the generation of all blood cell lineages. Obviously, the expansion of LTHSCs requires extensive self-renewal divisions. Since the experimental identification of blood stem/progenitor cells in the 1960s (11), the definition of cell culture conditions to maintain and expand LT-HSCs has become the quest for the holy grail. So far, all reported protocols have failed to reproducibly and reliably achieve the expansion of LT-HSCs with full blood cell reconstitution potential or only have provided a marginal advantage over existing conditions (12). Nevertheless, there is a tremendous effort world-wide in finding these optimized conditions. 
Now Adam Wilkinson and coauthors from the Nakauchi laboratory at Stanford University in collaboration with Tokyo University (Satoshi Yamazaki) reported that they have cracked the enigma (13). They have systematically tested and improved previous culture conditions and rationally altered factors according to the current knowledge of LTHSC behavior. To read-out increased LT-HSC numbers and/or increased potency, the authors went the cumbersome but ultimately required way of long-term competitive blood repopulation assays in recipient mice. They describe an astonishing LT-HSC net expansion from 50 freshly isolated input murine LT-HSCs to 12,000 LT-HSCs after 28 days in culture. They showed that the number of LT-HSCs can be further expanded in 57 days of culture, and that the cultured LT-HSCs are very potent to even engraft non-irradiated recipients. By lowering the cytokine concentration of stem cell factor (SCF) to $10 \mathrm{ng} / \mathrm{mL}$ in serum-free medium, they could prevent the excessive internalization of c-Kit as seen with higher concentrations. During in vitro culture, LTHSCs differentiate into blood cells of various differentiation states and lineages, which produce themselves paracrine soluble factors (e.g., cytokines, chemokines) and provide cell-cell interactions influencing the remaining immature pool of LT-HSCs. The continuous exchange of the medium to deplete these differentiation inducing factors has been shown beneficial for prolonged LT-HSC cultures in the past (14). Again, the authors here used a simple full medium exchange every three days, which proofed to be superior over half-medium exchanges in respect of maintaining transplantable repopulating LT-HSCs. The use of fibronectin-coated plates further increased the potency of cultured LT-HSCs in their blood cell reconstitution, in comparison to cell-culture-treated plastic, or collagen and gelatin coating. Surprisingly, they found that human serum albumin (HSA), which is supplemented in most serum-free media, had a detrimental effect on stem cell maintenance. They tested eleven other substitutes for albumin, which have been reported to support various cell cultures and revealed that polyvinyl alcohol (PVA), which is the main component of glue for children, can robustly substitute for HSA. LT-HSCs cultured in serum-free medium with PVA instead of HSA demonstrated a four-fold increase in blood reconstitution in primary recipient mice and a high and stable blood cell chimerism in secondary recipients, whereas LT-HSCs cultured in HSA-containing medium showed a successive decline of blood cell chimerism in primary recipients and completely failed to significantly reconstitute secondary recipients.
Combining all these improved culture conditions, the authors impressively showed a 50 to 200 -fold net increase in murine LT-HSCs with long-term blood reconstitution potential at day 28 of culture. Single LT-HSC cultures further demonstrated the real expansion of LT-HSCs (selfrenewal), although with variable efficacy. Most remarkably, the number and potency of in vitro expanded LT-HSC enabled robust engraftment and blood reconstitution in unconditioned recipient mice, an achievement unreachable by using equivalent fresh LT-HSCs. The authors briefly described the benefit of their improved culture conditions also for the long-term culture and expansion of human HSCs, which they documented in first experiments. However, a general applicability for human HSCs awaits future extended studies. The advanced culture conditions described by Wilkinson et al. do not require sophisticated coculture systems with feeder cells, genetic manipulation, or advanced technical devices. All components can be provided in compliance with good-manufacturing practice (GMP) for the fast translation into clinical application.

The authors provide some molecular explanations why their improved conditions are so much superior over previous protocols. However, the mechanism how the various factors interplay to enhance self-renewal of LTHSCs remains elusive. Though the seemingly simplicity of the cell culture protocol, its robustness requires further confirmations by other groups to become the new standard in the field. Other human HSC expansion protocols using small molecule inhibitors such as StemRegenin 1 (SR1) (15), UM171 (16), and valproic acid (VPA) $(17,18)$ as well as biologicals such as prostaglandin E2 $(19,20)$, or Insulingrowth factor-2 and Angiopoietin-1 (21) have entered clinical trials, and the outcomes of their benefit in medical use are awaited. It will be interesting to see whether some of these small molecules will further boost the described effects when combined with the improved conditions. How is the net expansion of LT-HSCs achieved? At a single LTHSC level it will be intriguing to see, whether LT-HSCs do divide as fast as downstream progenitors in these culture conditions, but retain their stem cell potential, and whether they divide more symmetrically to generate two stem cells. Furthermore, it remains unclear whether the LT-HSC pool is enlarged only in the first days of the improved in vitro culture, and whether the LT-HSCs enter quiescence afterwards. Alternatively, the LT-HSC generation time may be long but constant over the whole 57 days culture. The authors tested the blood reconstitution ability of the in vitro expanded LT-HSCs rigorously. However, questions remain 
about the molecular and metabolic properties of these LTHSCs. Are these LT-HSCs equally potent in multilineage reconstitution on a cell-to-cell comparison with freshly isolated LT-HSCs? Do the expanded LT-HSCs resemble the gene expression profile of freshly isolated dormant or activated LT-HSCs (22)? And are the genetic and epigenetic profiles of these LT-HSCs preserved over the whole culture period, considering that LT-HSC divisions may enforce genomic instability (23)? Many questions need to be addressed in the future to consolidate these exciting findings for the long-sought goal of human LT-HSC expansion in culture.

\section{Acknowledgments}

The author thanks Katharina Kiefer and Shakhawan Mustafa for critically reading the manuscript.

Funding: The study was supported by the Deutsche Forschungsgemeinschaft (SFB 834 Project Z1, the Excellence Cluster Cardiopulmonary Systems, and project RI2462/1-1) and the LOEWE Center Frankfurt Cancer Institute [Hessisches Ministerium für Wissenschaft und Kunst (III L 5-519/03/03.001-(0015)].

\section{Footnote}

Conflicts of Interest: The author has no conflicts of interest to declare.

Ethical Statement: The author is accountable for all aspects of the work in ensuring that questions related to the accuracy or integrity of any part of the work are appropriately investigated and resolved.

\section{References}

1. Copelan EA, Chojecki A, Lazarus HM, et al. Allogeneic hematopoietic cell transplantation; the current renaissance. Blood Rev 2019;34:34-44.

2. McRae HM, Voss AK, Thomas T. Are transplantable stem cells required for adult hematopoiesis? Exp Hematol 2019;75:1-10.

3. Rieger MA, Schroeder T. Hematopoiesis. Cold Spring Harb Perspect Biol 2012. doi: 10.1101/cshperspect. a008250.

4. Wilson A, Laurenti E, Oser G, et al. Hematopoietic stem cells reversibly switch from dormancy to self-renewal during homeostasis and repair. Cell 2008;135:1118-29.
5. Beerman I, Luis TC, Singbrant S, et al. The evolving view of the hematopoietic stem cell niche. Exp Hematol 2017;50:22-6.

6. Ito K, Ito K. Hematopoietic stem cell fate through metabolic control. Exp Hematol 2018;64:1-11.

7. Spangrude GJ, Heimfeld S, Weissman IL. Purification and characterization of mouse hematopoietic stem cells. Science 1988;241:58-62.

8. Laurenti E, Göttgens B. From haematopoietic stem cells to complex differentiation landscapes. Nature 2018;553:418-26.

9. Walasek MA, van Os R, de Haan G. Hematopoietic stem cell expansion: Challenges and opportunities. Ann N Y Acad Sci 2012;1266:138-50.

10. Thalheimer FB, Wingert S, De Giacomo $P$, et al. Cytokine-regulated GADD45G induces differentiation and lineage selection in hematopoietic stem cells. Stem Cell Reports 2014;3:34-43.

11. Till JE, Mcculloch EA. A direct measurement of the radiation sensitivity of normal mouse bone marrow cells. Radiat Res 1961;14:213-22.

12. Li Z, He XC, Li L. Hematopoietic stem cells: Self-renewal and expansion. Curr Opin Hematol 2019;26:258-65.

13. Wilkinson AC, Ishida R, Kikuchi M, et al. Long-term ex vivo haematopoietic-stem-cell expansion allows nonconditioned transplantation. Nature 2019;571:117-21.

14. Csaszar E, Kirouac DC, Yu M, et al. Rapid expansion of human hematopoietic stem cells by automated control of inhibitory feedback signaling. Cell Stem Cell 2012;10:218-29.

15. Boitano AE, Wang J, Romeo R, et al. Aryl hydrocarbon receptor antagonists promote the expansion of human hematopoietic stem cells. Science 2010;329:1345-8.

16. Fares I, Chagraoui J, Gareau Y, et al. Cord blood expansion. Pyrimidoindole derivatives are agonists of human hematopoietic stem cell self-renewal. Science 2014;345:1509-12.

17. Chaurasia P, Gajzer DC, Schaniel C, et al. Epigenetic reprogramming induces the expansion of cord blood stem cells. J Clin Invest 2014;124:2378-95.

18. Bug G, Gül H, Schwarz K, et al. Valproic acid stimulates proliferation and self-renewal of hematopoietic stem cells. Cancer Res 2005;65:2537-41.

19. North TE, Goessling W, Walkley CR, et al. Prostaglandin E2 regulates vertebrate haematopoietic stem cell homeostasis. Nature 2007;447:1007-11.

20. Hoggatt J, Mohammad KS, Singh P, et al. Prostaglandin E2 enhances long-term repopulation but does not 
permanently alter inherent stem cell competitiveness. Blood 2013;122:2997-3000.

21. Zhang CC, Lodish HF. Murine hematopoietic stem cells change their surface phenotype during ex vivo expansion. Blood 2005;105:4314-20.

22. Cabezas-Wallscheid N, Buettner F, Sommerkamp P,

Cite this article as: Rieger MA. How children's glue fixes a decades old enigma. Ann Transl Med 2019;7(Suppl 8):S348. doi: 10.21037/atm.2019.09.90 et al. Vitamin A-Retinoic Acid Signaling Regulates Hematopoietic Stem Cell Dormancy. Cell 2017;169:80723.e19.

23. Walter D, Lier A, Geiselhart A, et al. Exit from dormancy provokes DNA-damage-induced attrition in haematopoietic stem cells. Nature 2015;520:549-52. 